\title{
Real-time surveillance against COVID-19 is needed in Europe
}

\author{
yoshiyasu takefuji ${ }^{1}$ \\ ${ }^{1}$ Affiliation not available
}

October 15,2020

N. W. Ruktanonchai et al. assessed the impact of coordinated COVID-19 exit strategies across Europe (1). Their recommendation emphasized the effect of community coordination of nonpharmaceutical interventions (NPIs). NPIs are similar to community mitigation strategies (CMSs). Data of Europe shows the resurgence of the number of deaths due to COVID-19 reaching 1000 deaths per day. Europe needs the stronger quarantine monitoring for COVID-19 exit strategies.

According to Worldometer, as of Oct. 8 in 2020 the number of deaths due to the COVID-19 in the United States per day is around 500 per day so that the strong surveillance fence against COVID-19 is needed in order to exit from the COVID-19 pandemic. According to Statista as shown in Fig. 1, Fig. 1 shows the resurgence of the number of deaths in Europe due to COVID-19 reaching 1000 deaths per day. Relaxing lockdown together may immediately spread the COVID-19 pandemic again.

In order to relax the lockdown in Europe, the best effective surveillance should be implemented among surveillances in many countries (2).

Hsiao-HuiTsou et al. showed three mathematical strategies and analyzed their models: Strategy A: lack of prevention of subclinical transmission, Strategy B: partial prevention using testing with different accuracy, and Strategy C (Taiwan policy): complete prevention by isolating all at-risk people (3). They recommended the complete prevention strategy of Taiwan.

Data from Worldometer as of Oct. 72020 revealed that the cumulative number of deaths due to COVID-19 in Taiwan is only 7 while that of Europe is 235310 as of Oct. 52020 according to ECDC. The number of deaths in Europe is often over 500 per day as shown in Fig.1. Therefore, Europe needs the stronger quarantine monitoring which Taiwan has been operating in order to relax NPIs or CMSs. 


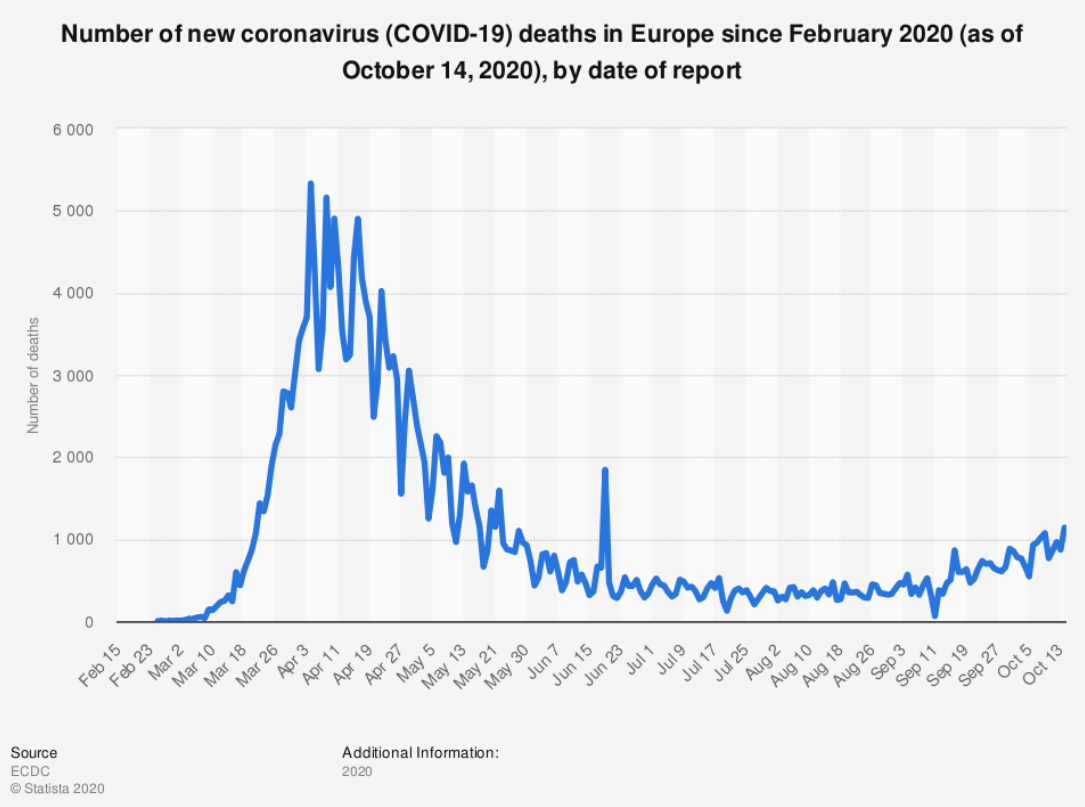

Fig. 1 the number of deaths due to COVID-19 in Europe

Source: Statista 2020

https://www.statista.com/statistics/1102288/coronavirus-deaths-development-europe/

Taiwan's contact-tracing and quarantine monitoring is a GPS-based information system called Intelligent Electronic Fences System (IEFS) (4). If GPS information is not available, based on an individual's mobile phone signals and nearby cell towers, it triangulates the location of quarantined individuals (4). It monitors the nation's entire quarantined population and any potential people that they may come into contact with. It tracks them in real time and retrospectively for up to a month (4). Taiwan is tracking 55,000 People under home quarantine in real time (5).

Complete prevention by isolating all at-risk people plays a key role in mitigating the COVID-19 pandemic for COVID-19 exit strategies in Europe.

This manuscript has not been published and is not under consideration for publication elsewhere. There is no fund in this research. The author has no conflict of interest. The author has read the manuscript and has approved this submission.

References:

1. N. W. Ruktanonchai et al., Assessing the impact of coordinated COVID-19 exit strategies across Europe, Science 18 Sep 2020: Vol. 369, Issue 6510, pp. 1465-1470 DOI: 10.1126/science.abc5096

2. Couch Danielle L et al., COVID-19-Extending Surveillance and the Panopticon, Journal of bioethical inquiry, 1-6. 25 Aug. 2020, doi:10.1007/s11673-020-10036-5

3. Hsiao-HuiTsou et al., The effect of preventing subclinical transmission on the containment of COVID-19: Mathematical modeling and experience in Taiwan, Contemporary Clinical Trials, Vol 96, 2020https://doi.org/10.1016/j.cct.2020.106101.

4. Po-Chang Lee et al., What we can learn from Taiwan's response to the covid-19 epidemic, July 21, 2020, https://blogs.bmj.com/bmj/2020/07/21/what-we-can-learn-from-taiwans-response-to-the-covid-19-epidemic 
5. CECC Reports No New Confirmed Cases; 408 Patients Released from Isolation, Taiwan Centers for Disease Control (Taiwan CEC) (May 22, 2020),https://perma.cc/QR83-WPH4; Mary Hui, How Taiwan Is Tracking 55,000 People under Home Quarantine in Real Time, Quartz (Apr. 1, 2020),https://perma.cc/9LAF-C8M4 